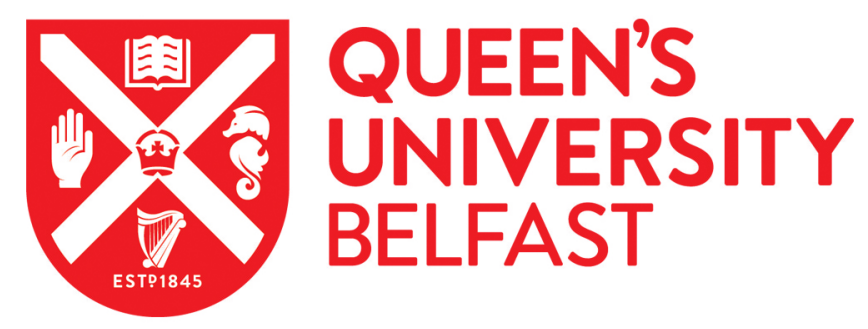

\title{
An evaluation of portion size estimation aids: Consumer perspectives on their effectiveness
}

\author{
Faulkner, G. P., Livingstone, M. B. E., Pourshahidi, L. K., Spence, M., Dean, M., O'Brien, S., Gibney, E. R., \\ Wallace, JMW., McCaffrey, TA., \& Kerr, MA. (2017). An evaluation of portion size estimation aids: Consumer \\ perspectives on their effectiveness. Appetite, 114, 200-208. https://doi.org/10.1016/j.appet.2017.03.027
}

\section{Published in:}

Appetite

\section{Document Version:}

Peer reviewed version

Queen's University Belfast - Research Portal:

Link to publication record in Queen's University Belfast Research Portal

\section{Publisher rights}

Copyright 2017 Elsevier.

This manuscript is distributed under a Creative Commons Attribution-NonCommercial-NoDerivs License

(https://creativecommons.org/licenses/by-nc-nd/4.0/), which permits distribution and reproduction for non-commercial purposes, provided the author and source are cited.

\section{General rights}

Copyright for the publications made accessible via the Queen's University Belfast Research Portal is retained by the author(s) and / or other copyright owners and it is a condition of accessing these publications that users recognise and abide by the legal requirements associated with these rights.

Take down policy

The Research Portal is Queen's institutional repository that provides access to Queen's research output. Every effort has been made to ensure that content in the Research Portal does not infringe any person's rights, or applicable UK laws. If you discover content in the Research Portal that you believe breaches copyright or violates any law, please contact openaccess@qub.ac.uk. 
Title: An evaluation of portion size estimation aids: consumer perspectives on their effectiveness

Authors: Gemma P. Faulkner ${ }^{1,5}$, M. Barbara E. Livingstone ${ }^{1}$, L. Kirsty Pourshahidi ${ }^{1}$, Michelle Spence $^{2}$, Moira Dean ${ }^{2}$, Sinead O’Brien ${ }^{3}$, Eileen R. Gibney ${ }^{3}$, Julie M.W.

Wallace $^{1}$, Tracy A. McCaffrey ${ }^{1.4}$ and Maeve A. Kerr ${ }^{1 *}$

\section{Institutions:}

${ }^{1}$ Northern Ireland Centre for Food and Health (NICHE), University of Ulster, Cromore Road, Coleraine, BT52 1SA.

${ }^{2}$ School of Biological Sciences, Queen's University Belfast, BT9 5AG, UK.

${ }^{3}$ Institute of Food and Health, University College Dublin, Belfield, Dublin 4, Ireland.

${ }^{4}$ Department of Nutrition and Dietetics, Monash University, Notting Hill, VIC 3168, Australia

${ }^{5}$ Life and Health Sciences, School of Science, IT Sligo, Ash Lane, Co. Sligo, Ireland

*Corresponding author: Dr Maeve A. Kerr

Address: Room W2030, School of Biomedical Sciences, University of Ulster, Coleraine campus, Cromore Road, Coleraine, Co. Londonderry, Northern Ireland, BT52 1SA.

Telephone: +442870123515

Fax: +442870123023

Email: ma.kerr@ulster.ac.uk

Running title: Perspectives of portion size estimation aids

Keywords: portion size, obesity, consumer, energy intake

\section{Acknowledgements}


The authors would like to acknowledge Miss Lorraine McGowan for helping with data collection.

\section{Financial support}

$\mathrm{PhD}$ sponsorship was obtained from the Department for Employment and Learning (DEL), Northern Ireland. This material is based on works supported by safefood, the Food Safety Promotion Board, under Grant number 07-2010. safefood had no role in the design, analysis or writing of this article.

\section{Conflict of interest}

None.

\section{Authorship}

G.P.F., M.B.E.L., M.A.K., T.A.M.C., J.M.W.W. and L.K.P. formulated the research questions and designed the study protocol. G.P.F. and L.K.P. carried out the study and analysed the data, G.P.F. wrote the manuscript; M.B.E.L., M.A.K, T.A.M.C., M.A.K, M.S., M.D, S.O.B. and E.R.G provided guidance on the analysis and write-up, and comment on drafts of the manuscript.

\section{Ethics Statement}

This study was conducted according to the guidelines laid down in the Declaration of Helsinki and all procedures involving human subjects were approved by the University of Ulster Research Ethics Filter Committee. 
Title: An evaluation of portion size estimation aids: consumer perspectives on their effectiveness

\begin{abstract}
Objective: This qualitative study aimed to investigate consumer opinions on the usefulness of portion size estimation aids (PSEA); consumer preferences in terms of format and context for use; and the level of detail of guidance considered necessary for the effective application of PSEA.
\end{abstract}

Design: Six focus groups (three to eight participants per group) were conducted to elicit views on PSEA. The discussions were recorded, transcribed verbatim and analysed by two independent researchers using a template approach.

Setting: The focus groups were conducted in 2013 by an experienced moderator in various sites across the island of Ireland (three in the Republic of Ireland and three in Northern Ireland) including local leisure, community and resource centres; the home environment; and a university meeting room.

Participants: General population, males $(n=17)$ and females $(n=15)$ aged $18-64$ years old. Participants were recruited from both urban and rural locations representing a range of socioeconomic groups.

Results: The majority of participants deemed the coloured portion pots and disposable plastic cup (household measures) to be useful particularly for the estimation of amorphous cereal products (e.g. breakfast cereals). Preferences were evident for "visual" PSEA (reference objects, household measures and food packaging) rather than 'quantities and measures' such as weighing in grams or ounces. Participants stated that PS education should be concise, consistent, from a reputable source, initiated at school age and communicated innovatively e.g. mobile app or TV advertisement. Guidance in relation to gender, age and activity level was favoured over a "one size fits all" approach.

Conclusions: This study identified consumer preferences and acceptance of "visual" PSEA such as portion pots/ cups to estimate appropriate PS of amorphous grain foods such as breakfast cereals, pasta and rice. Concise information from a reputable source in relation to gender, age and activity level should accompany PSEA. 
2 An increase in the availability of larger food portion sizes (PS) is one of many factors promoting a 3 positive energy balance amongst consumers and contributing to the escalating rates of overweight and obesity ${ }^{(1-2)}$. Although this phenomenon initiated in the $\mathrm{US}^{(3)}$, similar trends have been observed in the $\mathrm{UK}^{4}$ and Ireland ${ }^{(5)}$ in recent years. Larger PS are associated with higher energy intakes ${ }^{(6)}$, in both acute ${ }^{(7-12)}$ and longer term studies of up to one month ${ }^{(1)}$, conducted within and outside of the home ${ }^{(13-14)}$. Larger PS outside of the home have become "consumption norms" for consumers ${ }^{(15)}$, leading them to overestimate their PS in the home ${ }^{(13)}$. Furthermore, value deals (e.g. buy one get one free) can lead consumers to purchase more than they need ${ }^{(16)}$, in turn contributing to their distorted perceptions of appropriate PS.

The traditional reference to PS as "small, medium or large", particularly outside of the home e.g. in restaurants, fast food outlets and cinemas, may be too ambiguous and subjective for consumers ${ }^{(17-18)}$ and more specific guidance may be warranted. Although weighing scales and graduated measuring apparatus (e.g. jugs) are generally considered the most accurate portion size estimation aids (PSEA), they also tend to be the most burdensome and time consuming methods of measuring PS in the home ${ }^{(18)}$. There are various other aids available from different sources ranging from twodimensional aids such as food photographs to common household items such as the cup.

In recent years, national dietary guidelines including those from the US ${ }^{(19)}$ and Canada ${ }^{(20)}$ have communications. In the Republic of Ireland, the Food Pyramid and Healthy Eating Guidelines contain detailed guidance on appropriate SS for different sub-groups of the population ${ }^{(21)}$. In a related survey, a sample of consumers indicated their preference for the $200 \mathrm{ml}$ plastic cup over the dessertspoon for communicating SS of starchy foods, and the palm of the hand rather than the deck of cards for SS of meat, fish and alternatives ${ }^{(22)}$. Additional common household measures and reference objects i.e. the teaspoon and matchbox are also used by this guide to illustrate the SS of fats and oils, and cheese respectively. However, no evaluation of consumer opinions on the usefulness and acceptability these aids have been conducted to date. In contrast to the aforementioned countries, in the UK there is currently an absence of national quantitative guidance apart from that which is available for the fruit and vegetable food group ${ }^{23}$. 'The Eatwell Plate ${ }^{24}$ depicts the importance of a balanced diet, leaving SS determination to the discretion of consumers themselves. There are various SS guides available from non-governmental organisations (NGOs) and the food industry which tend to communicate inconsistent and sometimes conflicting advice 
which is confusing for consumers ${ }^{(25)}$. As a result, it has been suggested that 'The Eatwell Plate' should be accompanied by additional resources on food $\mathrm{SS}^{(26-28)}$.

Overall, despite the wide availability of various PSEA, there are limited data to date on consumer perceptions of the usefulness and their preferences for different types of PSEA which is of importance to inform effective public health campaigns in relation to guidance on appropriate PS. Therefore, the aims of the present study were to investigate consumer opinions on the usefulness of PSEA; consumer preferences in terms of format and context for use; and the level of detail of guidance considered necessary for the effective application of PSEA. A previous study evaluated (in practice) the precision, ease of use and likelihood of future use of a range of PSEA for various foods with diverse characteristics ${ }^{(29)}$.

Note for the purposes of this study the term PS refers to the amount of food intended to be consumed whereas SS refers to the amount of food recommended to be consumed e.g. in dietary guidelines or food labelling. However, it was apparent that the aids selected for this study were inconsistently used to either estimate PS or SS. Therefore, for the purposes of this study all aids were referred to as PSEA.

\section{Methods}

This study was conducted according to the guidelines laid down in the Declaration of Helsinki and all procedures involving human participants were approved by the University of Ulster Research Ethics Filter Committee. Written informed consent was obtained from all participants before commencing the study protocol.

\section{Portion size estimation aids}

A wide range of existing PSEA that could be used to aid the estimation of PS of a range of commonly eaten foods of diverse visual and physical properties were selected. These included tools utilised for amorphous (i.e. foods without a definite shape e.g. rice), liquid, solids, cooked, and dried foods. An extensive search was conducted for any PSEA that were available to the public; this included an online web search, review of household items and communication with dietitians, nutritionists and public health agencies. The range of available PSEA were grouped into four 
different categories: quantities and measures (e.g. SS in grams/millilitres on food labels), reference objects (e.g. a small matchbox for a SS of cheese), household measures and utensils (e.g. 200ml disposable cup and coloured portion pots from Rosemary Conley ${ }^{\mathrm{TM}}$ which included a guide detailing the colour of pot that corresponded with certain foods), and indicators on food packets (e.g. SS demarcations on the packaging of a block of cheddar cheese). Only PSEA which were relevant and available to Irish and UK consumers were chosen, others such as the baseball, which originates from the US, were not included. A full list of the PSEA (and their corresponding foods which were used in a previous study ${ }^{(29)}$ ) are presented in Table 1.

\section{Focus group recruitment}

Participants were recruited via a convenience sampling method using email distribution lists (sent to University staff and students) and social media advertisements. Recruitment opportunities were also sought in local community/volunteer groups and town centres between January and March 2013. Efforts were made to recruit from city, urban and rural locations, representing both males and females as well as a range of age groups, educational backgrounds and/or socio-economic groups, in order to capture a wide range of views. Inclusion criterion was that participants must be aged 1864 years old. Participants with similar characteristics (such as age, gender, occupational status) were grouped together as it was felt this might facilitate interaction amongst them. Six focus groups (3 in Northern Ireland and 3 in the Republic of Ireland) with three to eight participants per focus group were formed with 32 participants in total (17 males; 15 females, aged 18-64 years) (Table 2). Characteristics of all participants are included in Table 3. The focus groups were conducted by an experienced moderator (G.P.F.) in informal confidential settings familiar to the participants including local leisure, community and resource centres; the home environment; and a university meeting room. Each focus group was audio recorded with the consent of participants and lasted approximately 60-90 minutes. Data on personal characteristics (self-reported weight and height; age category; special diets; occupational status, and household information) were collected using a brief self-administered questionnaire which also investigated participants' use of PSEA or intention to use PSEA pre and post focus group participation respectively. Recruitment ceased when data saturation was achieved such that no new themes were established in the data. A gift voucher to the value of $£ 10 / € 15$ was given to each participant as disturbance allowance. 
A semi-structured discussion guide with open-ended topics was designed following a literature review of consumer opinions on PSEA. Initially, consumer knowledge of PS/ SS and guidance to help with portion size estimation was explored; progressing to the exploration of consumer opinions on the usefulness and preferences for PSEA and related guidance. The specific issues explored were as follows:

- Understanding of PS and SS

- Current practice of PS control strategies (i.e. PSEA or other methods to control PS)

- Perceived need for SS guidance/ aids

- Perceived usefulness of PSEA (the PSEA were shown in the following order: quantities and measures, reference objects, household measures and utensils, and indicators on food packets)

- Preferences for PSEA: format i.e. specific PSEA from quantities and measures/ reference objects/ household measures and utensils/ indicators on food packets, level of detail of guidance accompanying the PSEA, context of use, preferred sources of information (e.g. government/ food industry) and specific foods/ food groups for consideration (additional materials were used as prompts i.e. the Food Safety Authority of Ireland's healthy eating food guide which includes the food pyramid and guides on SS specific to different age, gender and activity levels ${ }^{(30)}$ and the UK's 'Eatwell Plate' which guides on the proportion of foods from each food group ${ }^{(24)}$ ).

The full discussion guide is available as Supplementary Online Material. The PSEA were used as visual prompts to facilitate focussed discussion. The categories of PSEA were presented to participants in a uniform order, however, within each category the order of the tools presented was varied to eliminate order bias in the discussion. The moderator made every effort to seek opinions from all participants and encouraged elaboration on all discussion points, using probes if necessary to redirect or facilitate discussion. The semi-structured discussion guide was pilot tested in advance of data collection to ensure clarity and comprehension and refined prior to implementation.

\section{Analysis}

The audio recordings for all six focus groups were professionally transcribed verbatim and reviewed by the moderator for accuracy. The transcripts were uploaded to the qualitative data analysis software package NVivo 9 (QSR International Pty Ltd., Doncaster, Victoria, Australia. A template approach was used to analyse the transcripts ${ }^{(31)}$. Two independent researchers named G.P.F. and L.K.P., defined and described the codes based on the research questions. Five broad code categories formed the code template: understanding of PS/ SS; current practice of PS control 
strategies; investigating the perceived usefulness of SS guidance/ aids; opinions on PSEA; and overall views of PSEA. The transcripts were read repeatedly in order to achieve data immersion. To determine the applicability of the individual codes to interview transcripts, the two researchers independently applied the initial template of codes to two transcripts and compared the results, wherein a decision was made to use the predetermined code template. The remaining transcripts were coded in a similar manner, and, for corroboration purposes, the researchers discussed the fundamental nature of each category code. Both reviewers agreed that data saturation had occurred as no new themes emerged in the last two transcripts. Quotations from participants were extracted to illustrate typical themes, individual participants were not identified on the transcripts therefore the quotations were used to express the opinions of the minority and majority views within the groups. IBM SPSS (version 20) was used to analyse quantitative data from the participant characteristics questionnaire.

\section{Results}

\section{Participant characteristics}

Participants were mostly aged 26-45 years (47\%); of healthy weight (BMI $18.5-24.9 \mathrm{~kg} / \mathrm{m}^{2}$ ) (45\%); employed full-time (28\%), unemployed (22\%) or students $(22 \%)$; following no special diet (78\%); responsible for grocery shopping (44\%) and preparing/ cooking meals in the household (50\%). A complete overview of subject characteristics is included in Table 3 and a summary of key results is also included in Table 4.

Note, any reference within the results to the 'majority' or 'most' of the participants means more than $50 \%$ of participants; the' minority' or 'some' means less than $50 \%$ of participants.

\section{Understanding of portion size and serving size}

It was evident across all groups that there was a lot of confusion in terms of the interpretation of a PS versus a SS. Majority of participants were not able to correctly differentiate between the terms PS and SS i.e. PS being the amount of food intended to be consumed by an individual whereas SS is the amount of food that is recommended to be consumed by an individual ${ }^{(32)}$. While some 
participants reported that the terms had the same meaning, the majority reported that they were the opposite to the Institute of Grocery Distribution definitions outlined above ${ }^{(32)}$ :

"Serving size is the size of the plate in front of you. It could be anything. Portion means the recommended intake of the meal for an individual" (group 4, males aged 18-64 years).

"Yeah, serving size is what you give yourself, portion size is what you probably should give yourself” (group 6, females aged 18-35 years).

Some participants also questioned whether a SS was the total amount to be eaten per day. Nonetheless the majority were unanimous in thinking that SS was unrealistic and too small particularly for breakfast cereals:

“...on other brands of cereal it says 25 servings, and I would say you would get seven or eight out of it” (group 2, males aged 18-35 years).

"Well sometimes, this is what amuses me, you know they say something is supposed to be a meal for two, but you would eat it all yourself” (group 1, females aged 36-64 years).

\section{Current practice of portion size control strategies}

Within this theme, the discussion explored the participants' use and awareness different portion control strategies and selection methods. Majority of groups indicated that their PS is determined by habit or judged by eye, so their PS selection was very much based on what they were familiar with based on previous experiences. The PS of breakfast cereals in particular was determined by the size of the bowl "...you go by the look of the bowl”, or for other foods the PS was one whole piece e.g. one steak or chicken fillet.

\section{“...it's just what looks right in the pot” (group 2, males aged 18-35 years);}

Usually the amount that was cooked would be eaten. For those who were not usually involved in cooking or preparing meals, their PS was determined by the server and they would generally "clean the plate":

“...if the girlfriend is cooking for me, she puts it out there and I eat it all, without rhyme or reason. Probably twice as much!” (group 4, males aged 18-64 years). 
"I would eat until it was all gone. If there was too much meat I would just eat it until it was finished because then I can wash the plate otherwise there's a dirty plate...and that annoys me" (group 6, females aged 18-35 years).

Participants were previously aware of PS information from the media, food labels and slimming groups, however, they their awareness off these aids did not always translate into their application to help them with PS control and selection. Most participants were aware of the food pyramid and either 'The Eatwell Plate' 24 or a segmented diet plate. Only some participants had noticed SS on food labels or the comparison of reference objects such as the matchbox and hand physiology to PS. Participants were aware of other PSEA including the "spaghetti hole", baking spoons, chopping board with graduated ruler along the side, pre-portioned packaged foods and using handfuls or fists. Some of them reported use of a pot or a cup for foods like rice and porridge to determine the correct ratio of grain to liquid for cooking rather than portion control.

\section{Perceived need for serving size guidance/ aids}

Within this theme, the perceived need for SS guidance and PSEA was discussed as well as the participants' views on the importance of consideration for PS. Overall, most participants felt that PS guidance was needed and that PSEA would be useful, particularly at the stage of food preparation. In addition, there was a general consensus that PS was not considered to be important in certain instances such as when feeling extremely hungry. However, some groups were of the opinion that adhering to PS advice would be too regimented and that food type or a "balanced diet" was most important rather than specific amounts:

“Do you know, it's just because you buy a bag of crisps it doesn't mean to say you have to eat the whole packet although sometimes you do. Or the same with chocolate bars as well, I think one here and there doesn't do anybody any harm, as long as you balance it" (group 6, females aged 18-35 years).

On the other hand, there were some instances where participants felt that PS and PSEA were not of significant importance to them individually i.e. if they were of normal weight and in the younger age bracket. Specifically, participants were generally of the opinion that PS "should be important" to them but that PS and PSEA were really only for the concern of "dieters" i.e. those following a weight-loss diet: 
“I think it's just in general like, I know some people are going to be more like people who are doing specific diets like Weight Watchers or Slimming World or something like, going to be weighing all the time, but it's not going to apply like to everybody" (group 2, males aged 18-35 years).

“...they all seem to be intended for people who wish to limit their food and calculate calories precisely; whereas that's not something I ever wish to do, to count calories. It's about just getting an average meal every day” (group 4, males aged 18-64 years).

The younger males and females indicated that PS was not a concern for them at present but perhaps later in life. It was also indicated that the older age group should not be forgotten about when it comes to PS as they are "not as active":

"I think maybe not at the moment but as you get older it might become more important" (group 2, males aged 18-35 years).

Lastly, a minority of participants mentioned 'time' as a factor that influenced their consideration for PS, they indicated that they were too busy to take the time to use PSEA:

"If you had time on your hands but a lot of people in this day and age are out working and they are always on the go and don't have time to do all that...if you've got two babies screaming at you..." (group 6, females aged 18-35 years).

\section{Perceived usefulness of portion size estimation aids}

The perceived usefulness of the PSEA was considered in relation to the groupings of PSEA i.e. quantities and measures; reference objects; household measures; and indicators on food packets. By in large, the majority of participants were in favour of the reference objects, household measures and indicators on food packets but noted some refinements that may be necessary for their effective application:

Quantities and measures - Measuring and weighing out foods were thought to be too laborious and that it was only necessary when baking or following a recipe. However, the middle-aged females (group 3, females aged 36-64 years) said they may use the weighing scales on one occasion then subsequently judge the amount based on the initial measurement. It was also pointed out that older people generally do not use the metric system, they still think in terms of pounds and ounces rather 
than grams which have been more commonly used in recent years. They felt that SS on food labels may be useful for interpreting nutritional information.

Reference objects - The groups were by majority in favour of the reference objects but some felt that the size of them was too small and unrealistic compared to what they would eat. Some participants were confused by the food photographs and thought that they suggested individual foods were to be eaten in isolation and not as whole meals:

“Not for me, because I'd never just serve those things by themselves” (group 4, males aged 18-64 years).

“... I'd never have just chips by itself, and curry without rice” (group 4, males aged 18-64 years).

Household measures - In general, the groups liked the coloured portion pots and the disposable cup measure, they thought they were easy to use, particularly for amorphous grains and flour. It would help them cook the appropriate amounts and avoid food waste, however, some would be discouraged by the extra washing up. There was concern noted among the younger females (group 6, females aged 18-35 years) that the coloured portion pots would go unused and that the disposable cup was not very "eye-catching”. However, it was suggested to leave a disposable cup in food packets of dried foods such as rice to use as a "scoop" when needed (group 4, males aged 18-64 years). Opinions tended to deviate with regards to the spoons, with some indicating they would use one for condiments including jam and honey but others disagreed:

"I would put honey or if I had a tablespoon full of jam or something like that. You know salady stuff or...” (group 1, females aged 36-64 years).

“No, I don't think so not for peanut butter or jam and marmalade, or honey, you're not going to put it in the spoon to take it back off again to put it on the bread or whatever" (group 2, males aged 1835 years).

Indicators on food packets - The majority were not aware of indicators on food packaging before but thought they could be useful for some foods particularly those that need to be sliced or poured (e.g. cheese and rice respectively). It was highlighted that the markings on tinned foods were not a good idea, as once opened these foods were not usually stored in the tin. The groups liked the idea of the markings on the cheese block but were of the opinion that they may be inconvenient to use. It was thought that markings on food packets could be a "novelty" for children. It was suggested that printing circles on spaghetti and markings on pasta packaging may be useful indicators of SS. In 
terms of SS as fractions of pie-shaped foods, participants liked the idea but emphasized that they would generally eat a whole pizza to avoid wastage.

\section{Preferences for portion size estimation aids}

There was a clear preference for the "visual" PSEA particularly the portion pots, cups, reference objects and indicators on food packaging (fractions and transparent demarcations) as it was said to be "less hassle" and "quicker" as opposed to the "boring" quantities and measures. Participants also liked using other common household items like scoops and bowls (for cereal):

"So definitely the visual, yeah rather than the grams and ounces" (group 3, females aged 36-64 years).

It was often mentioned that PSEA would be useful for everyday foods mainly the starchy foods such as rice, pasta, cereals, porridge and potatoes, and foods that were difficult to control PS such as cheese; rather than fruit and vegetables for which consumption would be encouraged. However, views on PSEA for discretionary items or "junk" foods (i.e. snacks of high energy density such as crisps and chocolate) and condiments (e.g. mayonnaise) were equivocal:

“That's something I always struggle with, rice, never know, you end up just pouring, pouring, then you could feed a family and then you end up dumping most of it" (group 2, males aged 18-35 years).

"It's okay saying your cheese should be that size, but then you put it in the fridge and you go back again and take another matchbox full out and eat it, you know, you can eat cheese all day. I love my cheese” (group 1, females aged 36-64 years).

“Well it says for fruit that more is better, so it doesn't make a difference really if you use that thing [cup] or not” (group 2, males aged 18-35 years).

Unanimously participants felt the PSEA should be provided to the public free of charge and that children in schools should be a prime target. SS guidance should be disseminated innovatively through a TV advertisement, fridge magnet, on food labels or a mobile phone app:

"An app would be good, a portion size app, download this is the size of toast you are meant to have in the morning or lasagne or this is the size of your cereal that we should have" (group 6, females aged 18-35 years). 
In terms of the format, there was some dislike for leaflets with the idea that they were "old" and "boring" but the format and colour coding of the Irish Healthy Eating Guidelines ${ }^{30}$ were well liked. It was suggested to have a short guide for the general public and a more detailed guide for those on special diets. Majority of participants felt it important to include a range of the number of daily SS. The idea of guidance segregated into age, gender and activity levels was appealing to most participants rather than a 'one size fits all' approach:

"I think I quite like the way that goes up... a person that exercises to a person that doesn't do much exercise and things like that. I think it's quite a good idea, going up in age groups and that as well" (group 6, females aged 18-35 years).

It was clear that all participants wanted the PSEA and guidance to come from one "reputable" source i.e. either government or a recognised public health authority and this should be followed consistently by all stakeholders particularly industry as there was some scepticism and distrust towards the food industry and their motives:

“...then the packaging would have to follow something that's authorized... because if there isn't one baseline, you know, how do you interpret where information, $X$ maybe on this, if they use one source of authority or information being on another packet which uses another" (group 1, females aged 36-64 years).

“...I don't think I'd trust it as much if it came from the industry, because they really want you to eat as much as you can... (group 4, males aged 18-64 years).

\section{Discussion}

Larger food PS is a factor that has been linked with higher energy intakes ${ }^{(6)}$ contributing to the current rates of overweight and obesity ${ }^{(1-2)}$. Previous research suggests that reference to PS as "small, medium or large", may be unclear ${ }^{(17-18)}$. Despite consumers' difficulty in controlling their food PS, there has been little research to evaluate their perceived usefulness of PSEA and their preferences for such PSEA. In Ireland, consumers preferred the idea of a cup over a dessertspoon for SS of starchy foods, and the palm of the hand rather than the deck of cards for SS of meat, fish and alternatives ${ }^{(22)}$, although, no evaluation of the perceived usefulness and acceptability of these PSEA has been conducted to date. 
In the present study, focus groups and a brief questionnaire were used to explore consumer opinions on the usefulness of PSEA; consumer preferences in terms of format and context for use; and the level of detail of guidance which would be necessary for their utilisation. The qualitative data which were collected conveyed a clear preference and acceptance (in theory) for "visual" PSEA (reference objects, household measures and food packaging) rather than quantities and measures i.e. weighing or measuring in grams/ millilitres/ ounces. In particular, amorphous grains including breakfast cereals, pasta and rice; and cheese were highlighted as foods for which PSEA may be most useful. The PSEA would most likely be used when preparing the main meal in the home. They were deemed necessary mainly for those on a weight loss diet or for older adults. One general consensus was that PS guidance should be concise, consistent, realistic, initiated at school age, from a reputable source, and communicated innovatively e.g. mobile app or TV advertisement. Guidance in relation to gender, age and activity level was favoured over a "one size fits all" approach.

In accordance with previous research, PSEA are viewed as being particularly useful for amorphous grain foods such as breakfast cereals, pasta and rice ${ }^{33}$, this previous study was conducted in the UK using focus groups also. Participants indicated that they find it particularly difficult to estimate PS of such foods. Similarly some participants expressed difficulty in controlling the amount of cheese they consume, consequently, stating that a PSEA may be useful. This is in agreement with another UK report that recommended the use of PSEA for foods high in saturated fat such as cheese ${ }^{28}$. The mixed opinions towards the need for PSEA for indulgent foods of high energy density e.g. chocolate and mayonnaise, in the current study were also reflective of previous findings ${ }^{33}$. The lack of interest in PSEA for these high energy dense foods is an issue which needs further exploration in future research. Researchers in the US who used a novel approach by inserting coloured potato chips at regular intervals in a tube of potato chips, found that consumption decreased by over $50 \%$ due to the segmentation cues which prompted a somewhat automatic subconscious response from consumers $^{34}$. The latter study may provide further scope for the development and promotion of indicators on food packets as participants were generally receptive to these as PSEA but reported issues with their usability. Another option to be explored in future research are pre-portioned packs of high energy dense foods, however, these tend to be more expensive than the larger value packs. Therefore, a more proportionate pricing system may make pre-portioned foods more acceptable to consumers. Nonetheless, the majority of PSEA considered in this study would generally evoke a conscious response from the reflective system of the brain, it may be interesting to explore more cues that could be subconsciously used by consumers such as the indicators on food packaging or the segmentation cues within food packets in future research. 
360

361

362

363

364

365

366

367

368

369

370

371

372

373

374

375

376

377

378

379

380

381

382

383

384

385

386

387

388

389

390

391

392

As current portion size selection tends to stem from both habit and tradition ${ }^{(33,35)}$, effectively communicating the benefits of adhering to more appropriate PS could be instrumental to instigating behavioural change ${ }^{36}$. Participants indicated that they currently judge their PS either through habit, by eye, using bowl size for items like breakfast cereals or in units for items like chicken breasts. Participants were generally acquiescent to PSEA although in some instances deemed them only necessary for those on a diet who wish to limit their intake of particular foods. There was a general consensus that PSEA were unnecessary for fruit and vegetables; participants did not consider the fact that PSEA could facilitate the '5-a-day' recommendation. Some stated they would be too busy to implement portion control while others said it would "make you crazy" using PSEA every day. The present study has alluded to initiating PS education in schools to instil appropriate habits at an early age. This may be a strategy to make PS education more amenable to children and younger adults. This finding also implicates future policy with regards children's nutrition as schools and educators could be a key intervention point in terms of reducing children's energy intake.

Further development is needed to incorporate PS guidance into more innovative communication formats. For example, in the US a multimedia approach is adopted including the use of mobile phone apps and a range of online resources and printed materials are provided ${ }^{(19,37)}$. Although there has been research into the use of such methods for dietary assessment, there are limited data on their use as PSEA pre consumption. A recent intervention conducted in the Netherlands evaluated a web based portion size tool and found it to be effective in raising awareness of recommended SS and overeating triggers from larger PS $^{(38)}$. In the current study, participants' were receptive to using the PSEA for food preparation in the home, suggesting that guidance on appropriate amounts of food to purchase (e.g. meat) or cook (e.g. rice) may be most effective at helping consumers to serve out and ultimately consume more appropriate PS. This implies that a key intervention point for policy makers in terms of PSEA may be pre-consumption, therefore it may be worthwhile communicating PSEA guidance in terms of raw/ pre-cooked amounts in future to encourage this practice. Perhaps estimation of PS while purchasing and preparing food may help to eliminate the habit of "cleaning the plate". In any case, it is imperative that future research considers the incorporation of PS guidance and PSEA into multi-media such as mobile phone apps, so that policy makers can effectively integrate PSEA into such mediums in order to innovatively target a wide range of consumers.

Age and gender differences were apparent with regards to preferences for PSEA. For example, the middle aged females (group 1) were more accepting of the idea of using spoons to aid them with portion control for condiments. On the other hand, the males of all ages indicated that they would 
not be likely to use spoons, a reason for this is that it would result in more washing-up. Males also indicated the unlikelihood of them using portion control for foods like cheese, this was illustrated in group 4 "I'd rather have it once a week and have a nice amount". This is a novel finding of the current study as age and gender differences were not apparent in a previous study of similar nature $^{33}$. Therefore, it is apparent that age and gender may need to be taken into consideration when communicating portion control guidance in future.

This study has reiterated consumers' distrust and lack of confidence in the food industry ${ }^{25}$ owing to the perception that they have 'ulterior motives. This gives clear virtue to consumers' desire for consistent guidance from a reputable source such as government or a public health authority, notwithstanding the fact that all advice should be non-prescriptive and serve to empower the consumer to make their own informed choices ${ }^{33}$. Successful public health initiatives such as the salt reduction campaign, involve facilitating the food industry to commit to consistency, transparency and standardisation for the benefit of the consumer. Adopting a similar approach with respect to food PS may be warranted so that guidance from all stakeholders is uniform. This study has also highlighted consumer confusion of the terms PS vs. SS, if 'one size fits all', and whether it's a "daily amount" 25 . Therefore, clear and concise supplementary information should be made available with the PSEA to clarify these issues.

A limitation of the present study is that drinks were not considered in relation to consumer preferences for PSEA. This was partly due to the fact that a previous study alluded to the fact that drinks were generally not considered by consumers in terms of PS estimation ${ }^{33}$, and secondly because there were limited PSEA available for drinks to consider as part of this research. Therefore, there is an opportunity to develop novel PSEA for drinks and to investigate whether these would be feasible for consumers to use.

\section{Conclusion}

This study has identified consumer preferences and acceptance for "visual" PSEA such as reference objects, household measures and indicators on food packaging. In particular, these were deemed to be most useful for amorphous grain foods such as breakfast cereals, pasta and rice. The following 4 key recommendations can be derived from this research.

1. PS education should be ingrained at a young age and disseminated through modern technologies to engage with the wider public. 
2. A concise, consistent, realistic and unified approach to PS guidance involving all stakeholders is warranted in order to gain consumer trust.

3. The current findings should be considered in conjunction with further research which examines the practical use and precision of such aids ${ }^{(29)}$.

4. The scope of the current research could be expanded to explore the feasibility of more recently developed PSEA available electronically via mobile phone apps or online. 


\section{References}

1. Jeffery RW, Rydell S, Dunn, CL et al. (2007) Effects of portion size on chronic energy intake. Int J Behav Nutr Phys Act 4, 27.

2. Kelly MT, Wallace JMW, Robson PJ et al. (2009) Increased portion size leads to a sustained increase in energy intake over $4 \mathrm{~d}$ in normal-weight and overweight men and women. $\mathrm{Br} J \mathrm{Nutr}$ 102, 470-477.

3. Smiciklas-Wright H, Mitchell DC, Mickle SJ et al. (2003) Foods commonly eaten in the United States, 1989-1991 and 1994-1996: are portion sizes changing? J Am Diet Assoc 103, 41-47.

4. Church S (2008) Trends in portion sizes in the UK - A preliminary review of published information. UK: Food Standards Agency.

5. McCarthy S, Robson P, Livingstone M et al. (2006) Associations between daily food intake and excess adiposity in Irish adults: towards the development of food-based dietary guidelines for reducing the prevalence of overweight and obesity. Int J Obes 30, 993-1002.

6. Rolls B, Roe L \& Meengs J (2006) Larger portion sizes lead to a sustained increase in energy intake over 2 days. J Am Diet Assoc 106, 543-549.

7. Diliberti N, Bordi PL, Conklin MT et al. (2004) Increased portion size leads to increased energy intake in a restaurant meal. Obes Res 12, 562-568.

8. Rolls BJ, Roe LS, Kral TVE et al. (2004a) Increasing the portion size of a packaged snack increases energy intake in men and women. Appetite 42, 63-69.

9. Rolls BJ, Roe LS, Meengs JS et al. (2004b) Increasing the portion size of a sandwich increases energy intake. J Am Diet Assoc 104, 367-372.

10. Flood JE, Roe LS \& Rolls BJ (2006) The effect of increased beverage portion size on energy intake at a meal. J Am Diet Assoc 106, 1984-1990.

11. Raynor HA \& Wing RR (2007) Package unit size and amount of food: do both influence intake? Obesity 15, 2311-2319.

12. Burger KS, Fisher JO \& Johnson SL (2011) Mechanisms behind the portion size effect: visibility and bite size. Obesity 19, 546-551.

13. Nielsen SJ \& Popkin BM (2003) Patterns and trends in food portion sizes, 1977-1998. J Amer Med Assoc 289 450-453.

14. Condrasky M, Ledikwe JH, Flood JE et al. (2007) Chefs' opinions of restaurant portion sizes. Obesity 15, 2086-2094.

15. Wansink B \& van Ittersum K (2007) Portion size me: downsizing our consumption norms. $J$ Am Diet Assoc 107, 1103-1106. 
16. Vermeer WM, Steenhuis IHM \& Seidell JC (2010) Portion size: a qualitative study of consumers' attitudes toward point-of-purchase interventions aimed at portion size. Health Educ Res 25, 109-120.

17. Young LR \& Nestle M (1998) Variation in perceptions of a 'medium' food portion: implications for dietary guidance. J Am Diet Assoc 98, 458-459.

18. Wrieden WL \& Momen NC (2009) Workshop 3: Novel approaches for estimating portion sizes. Eur J Clin Nutr 63, S80-S81.

19. U.S. Department of Agriculture (2011) ChooseMyPlate. Available from: http://www.choosemyplate.gov/index.html

20. Health Canada (2007) Eating well with Canada's Food Guide. Canada: Health Canada.

21. Food Safety Authority of Ireland (2011) Scientific recommendations for healthy eating in Ireland. Dublin: Food Safety Authority of Ireland.

22. Flynn MAT, O’Brien CM, Faulkner G et al. (2012) Revision of food-based dietary guidelines for Ireland, phase 1: evaluation of Ireland's food guide. Pub Health Nutr 15, 518-526.

23. National Health Service (2003) 5 A DAY portion sizes. Available from: http://www.nhs.uk/Livewell/5ADAY/Pages/Portionsizes.aspx

24. Food Standards Agency (2007) The Eatwell Plate. Available from: http://www.food.gov.uk/multimedia/pdfs/theeatwellplate.pdf

25. Faulkner GP, Pourshahidi LK, Wallace JMW et al. (2012) Serving size guidance for consumers: is it effective? Proc Nutr Soc 71, 610-621.

26. Anderson AS, Freeman J, Stead M et al. (2005) Consumer views on portion size guidance to assist adult dietary choices. Canada: Canadian Diabetes Association.

27. The British Dietetic Association (2007) Dietitians hail return of balanced diet plate. Birmingham: The British Dietetic Association.

28. Anderson AS, Barton K., Craigie A et al. (2008) Exploration of Adult Food Portion Size Tools. Scotland: NHS Health Scotland.

29. Faulkner GP, Kerr MA, Pourshahidi KP et al. (2016) An evaluation of portion size estimation aids: precision, ease of use and likelihood of future use. Pub Health Nutr 19, 2377-2387.

30. Food Safety Authority of Ireland (2012) Healthy eating and active living for adults, teenagers and children over 5 years - a food guide for health professionals and catering services. Dublin: Ireland.

31. Crabtree BF \& Miller WL (1999) Using codes and code manuals: a template organizing style of interpretation. In: Crabtree BF and Miller WL eds. Doing Qualitative Research. $2^{\text {nd }}$ ed. Newbury Park, California: Sage. 
497 32. Institute of Grocery Distribution (2008) Portion Size: A Review of Existing Approaches. 498 England: Institute of Grocery Distribution.

499 33. Institute of Grocery Distribution (2009) Portion size: understanding the consumer perspective. 500 England: Institute of Grocery Distribution.

501 34. Geier AB, Rozin P \& Doros G (2006) Unit bias - a new heuristic that helps explain the effect of 502 portion size on food intake. Psychol Sci 17, 521-525.

503 35. Spence M, Livingstone MBE, Hollywood LE, et al. (2013) A qualitative study of psychological, 504 social and behavioral barriers to appropriate food portion size control. Int J Behav Nutr Phys Act $505 \quad \mathbf{1 0}, 92$.

506 36. Elwood P \& Longley M (2010) My health: whose responsibility? A jury decides. J Epidemiol 507 Community Health 64, 761-764.

508 37. Daggett LM \& Rigdon KL (2006) A computer-assisted instructional program for teaching 509 portion size versus serving size. J Commun Health Nurs 23, 29-35.

510 38. Poelman MP, Steenhuis IHM, de Vet E, et al. (2013) The development and evaluation of an 511 internet-based intervention to increase awareness about food portion sizes: a randomised control 512 trial. J Nutr Educ Behav 45 (6), 701-707. 
514 Table 1 Foods (consider in the previous study ${ }^{29}$ ) and related portion size estimation aids

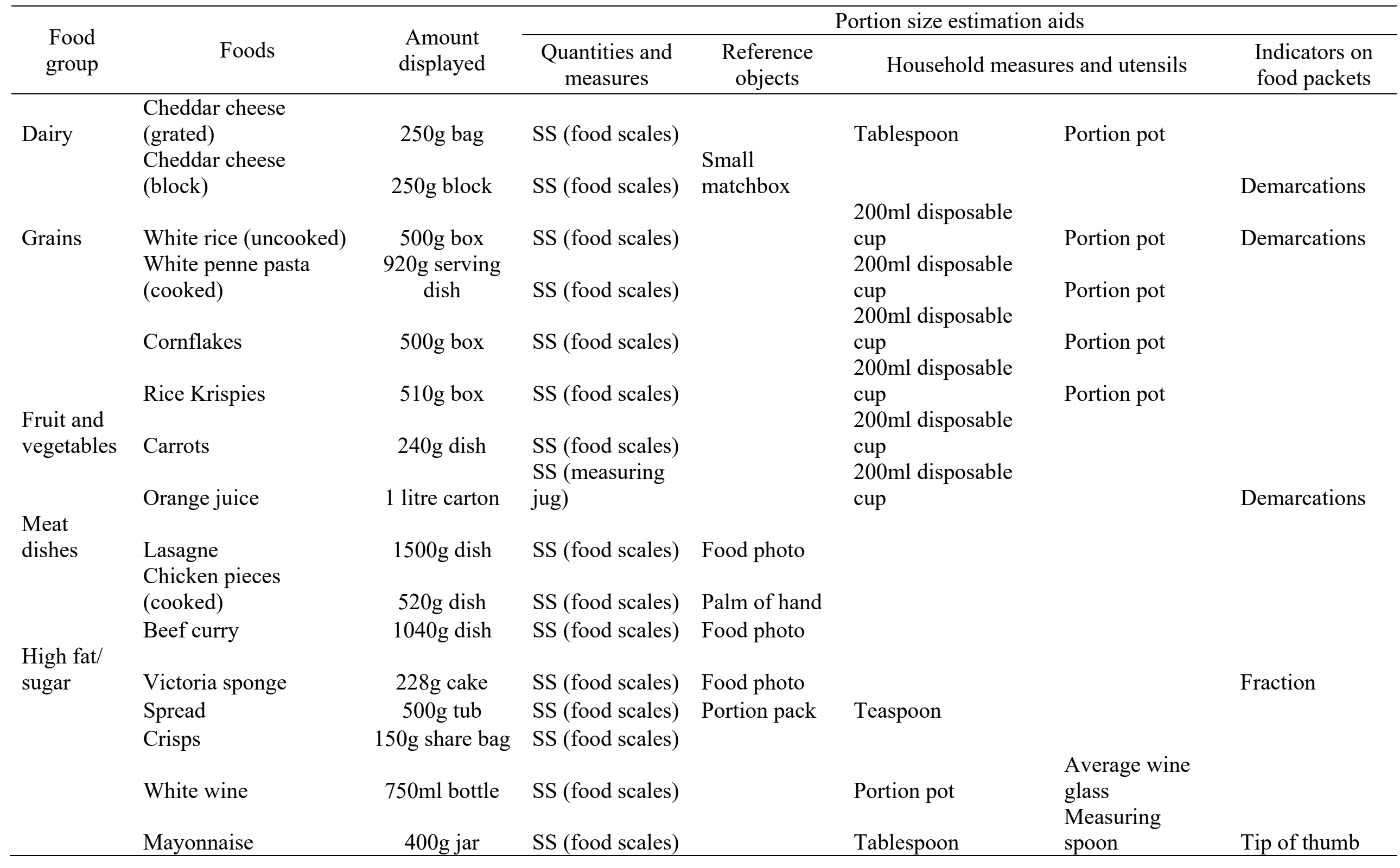


515 Table 2 Demographics of focus group participants

\begin{tabular}{ccccl}
\hline $\begin{array}{c}\text { Focus group } \\
\text { number }\end{array}$ & $n$ & Gender & Age range & \multicolumn{1}{c}{ Location } \\
\hline 1 & 5 & F & $36-64$ & NI - slimming group \\
2 & 5 & M & $18-35$ & ROI - community \\
3 & 7 & F & $36-64$ & ROI - community \\
4 & 8 & M & $18-64$ & ROI - university staff \\
5 & 4 & M & $18-64$ & NI - community \\
6 & 3 & F & $18-35$ & NI - community \\
\hline
\end{tabular}

NI, Northern Ireland; ROI, Republic of Ireland; F, female; M, male 
518 Table 3 Characteristics of the focus group participants ( $n$ 32)

$n$

Gender

$\begin{array}{ll}\text { Male } & 17 \\ \text { Female } & 15\end{array}$

Age (years)

$\begin{array}{lll}18-25 & 6 & 19 \\ 26-35 & 8 & 25 \\ 36-45 & 7 & 22 \\ 46-55 & 5 & 16 \\ 56-64 & 6 & 19\end{array}$

\section{BMI (kg/m2)*}

$\begin{array}{lll}\text { Normal weight (18.5-24.9) } & 13 & 45\end{array}$

Overweight (25.0-29.9) $\quad 10 \quad 34$

Obese $(\geq 30.0) \quad 61$

\section{Country of residence}

$\begin{array}{lll}\text { Northern Ireland } & 11 & 35\end{array}$

Republic of Ireland $\quad 20 \quad 65$

\begin{tabular}{lcc} 
Marital status & & \\
\hline Single & 11 & 34 \\
Living with partner & 14 & 13 \\
Married & 2 & 44 \\
Divorced/separated & 1 & 6 \\
Widowed &
\end{tabular}

\section{Highest level of education achieved}

Secondary (age 15/16 years) $\quad 2 \quad 6$

Secondary (age 17/18 years) $\quad 4 \quad 13$

$\begin{array}{lll}\text { Additional training (NVQ) } & 12 & 39\end{array}$

$\begin{array}{lll}\text { Undergraduate } & 6 & 19\end{array}$

$\begin{array}{lll}\text { Postgraduate } & 7 & 23\end{array}$

\section{Occupational status}

$\begin{array}{lll}\text { Employed FT } & 9 & 28\end{array}$

Employed PT $\quad 5 \quad 16$

$\begin{array}{lll}\text { FT home maker } & 7 & 22\end{array}$

$\begin{array}{lll}\text { Not employed } & 4 & 13\end{array}$

$\begin{array}{lll}\text { Student } & 7 & 22\end{array}$

\section{Smoking status/history}

$\begin{array}{lcc}\text { Current smoker } & 5 & 16 \\ \text { Ex smoker } & 8 & 25 \\ \text { Never smoked } & 19 & 59\end{array}$


Over the last year, have tried to:

$\begin{array}{lll}\text { lose weight } & 17 & 53 \\ \text { eat less fat } & 17 & 53 \\ \text { eat more fruit and vegetables } & 22 & 69 \\ \text { exercise more } & 23 & 72\end{array}$

Are you on a special diet?

$\begin{array}{lll}\text { No } & 25 & 78\end{array}$

Cholesterol lowering $\quad 1 \quad 3$

$\begin{array}{lll}\text { Slimming (self/prescribed) } & 6 & 19\end{array}$

How many people (inc. you) live in your household?

$\begin{array}{lcc}\text { Live alone } & 3 & 10 \\ \text { Two people } & 11 & 35 \\ \text { Three people } & 8 & 26 \\ \text { Four people } & 4 & 13 \\ \text { 5+ people } & 5 & 16\end{array}$

Are you responsible for grocery shopping?

Yes - I do most $\quad 14 \quad 44$

Yes - I am jointly responsible $\quad 11 \quad 34$

No-someone else does it $\quad 7 \quad 22$

Are you responsible for preparing/cooking meals?

$\begin{array}{lcc}\text { Yes - I do most } & 16 & 50 \\ \text { Yes - I am jointly responsible } & 11 & 34 \\ \text { No - someone else does it } & 5 & 16\end{array}$

$520 *$ Calculated from self-reported weight $(\mathrm{kg})$ and height $(\mathrm{m})$ 
521 Table 4 Summary of key focus group results by discussion theme

\begin{tabular}{|c|c|}
\hline $\begin{array}{l}\text { Current } \\
\text { understanding }\end{array}$ & $\begin{array}{l}\text { - Confusion evident between different terminology used } \\
\text { interchangeably/inconsistently (i.e. SS vs PS) } \\
\text { - Majority of participant unanimously agreed that current SS were } \\
\text { unrealistic and too small }\end{array}$ \\
\hline $\begin{array}{l}\text { Current PS/SS } \\
\text { practices }\end{array}$ & $\begin{array}{l}\text { - Current PS determined: } \\
\text { o "by habit" } \\
\text { o "judged by eye" } \\
\text { o using the size of the plate/bowl } \\
\text { o by the unit-size of foods, e.g. one steak or fillet } \\
\text { o by the individual responsible for cooking/serving the meal } \\
\text { - Awareness of PS information did not always translate into their use } \\
\text { - Use of PS control strategies was more commonly employed when } \\
\text { determining the amount to cook, particularly using household measures or } \\
\text { reference objects, e.g. a handful }\end{array}$ \\
\hline $\begin{array}{l}\text { Perceived need } \\
\text { for SS } \\
\text { guidance/aids }\end{array}$ & $\begin{array}{l}\text { - Most participants felt that PS guidance was needed particularly for when } \\
\text { preparing food } \\
\text { - This guidance should not override the importance of following a } \\
\text { "balanced diet" } \\
\text { - It was acknowledged that the importance of PS guidance would be } \\
\text { different depending on individual circumstances, e.g. age, body weight, } \\
\text { level of physical activity } \\
\text { - May be most relevant for "dieters" }\end{array}$ \\
\hline $\begin{array}{l}\text { Perceived } \\
\text { usefulness of } \\
\text { PSEA }\end{array}$ & $\begin{array}{l}\text { - Aids considered: } \\
\text { o Quantities and measures } \\
\text { o Reference objects } \\
\text { o Household measures } \\
\text { o Food packaging } \\
\text { - All types of PSEA were generally well-received by the majority of } \\
\text { participants, with the exception of the quantities and measures, which } \\
\text { were viewed as being too laborious } \\
\text { - Practical solutions and reference objects were perceived to be the most } \\
\text { useful }\end{array}$ \\
\hline $\begin{array}{l}\text { Preferences for } \\
\text { PSEA }\end{array}$ & 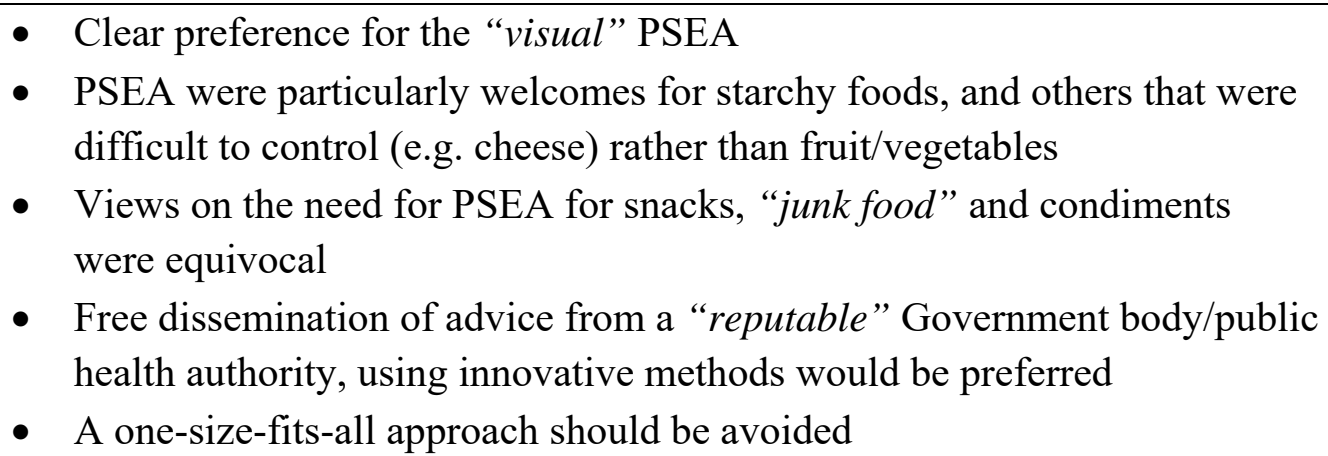 \\
\hline
\end{tabular}




\section{Supplementary Online Material}

524

525 1. Focus Group discussion guide (.pdf) uploaded separately.

526 2. Table 3 (full characteristics of all focus group participants) can be included as supplementary 527 online material at the discretion of the editor. 